\title{
Impact of morphological effects on the activity and stability of tungsten carbide catalysts for dry methane reforming
}

\author{
William P. Mounfield, III ${ }^{a}$ Aadesh Harale ${ }^{b}$, and Yuriy Román-Leshkov ${ }^{a *}$
}

a. Department of Chemical Engineering, Massachusetts Institute of Technology, Cambridge, Massachusetts 02139, USA b. Research \& Development Center, Saudi Aramco, Dhahran, KSA 31311

\section{Experimental Section}

Chemicals: Ammonium metatungstate hydrate (AMT, $\left.\left(\mathrm{NH}_{4}\right)_{6} \mathrm{H}_{2} \mathrm{~W}_{12} \mathrm{O}_{40} \cdot x \mathrm{H}_{2} \mathrm{O}\right)$, oleylamine (1-amino-9-octadecene) (70\% purity, SigmaAldrich), acetone, toluene, trimethylamine N-oxide (TANO), and 1,2 dodecanediol. All chemicals were used as received.

Thermogravimetric (TGA) analyses were performed with a TA Instruments Q500 analyzer equipped with a standard furnace (953032.901, TA Instruments) and a platinum pan (952018.906, TA Instruments). In a typical measurement, $25 \mathrm{mg}$ of sample was subjected to a heat ramp from $30{ }^{\circ} \mathrm{C}$ to $800{ }^{\circ} \mathrm{C}$ at a rate of $5^{\circ} \mathrm{C} / \mathrm{min}$ under a $5 \mathrm{~mL} / \mathrm{min}$ flow of air.

Table S1. Elemental composition and surface areas for pristine and spent catalysts.

\begin{tabular}{cccc} 
Catalyst & wt.\% - fresh & $\begin{array}{c}\text { Surface Area } \\
\left(\mathrm{m}^{2} / \mathrm{g}\right)-\text { fresh }\end{array}$ & $\begin{array}{c}\text { Surface Area } \\
\left(\mathrm{m}^{2} / \mathrm{g}\right)-\text { spent }\end{array}$ \\
\hline$\gamma-\mathrm{Al}_{2} \mathrm{O}_{3}$ & - & 216.0 & - \\
\hline $\mathrm{W}_{\mathrm{x}} \mathrm{C}-\mathrm{NP}$ & 9.8 & 204 & 5.2 \\
\hline $\mathrm{W}_{\mathrm{x}} \mathrm{C}-\mathrm{NR}$ & 9.5 & 188 & 147.2
\end{tabular}
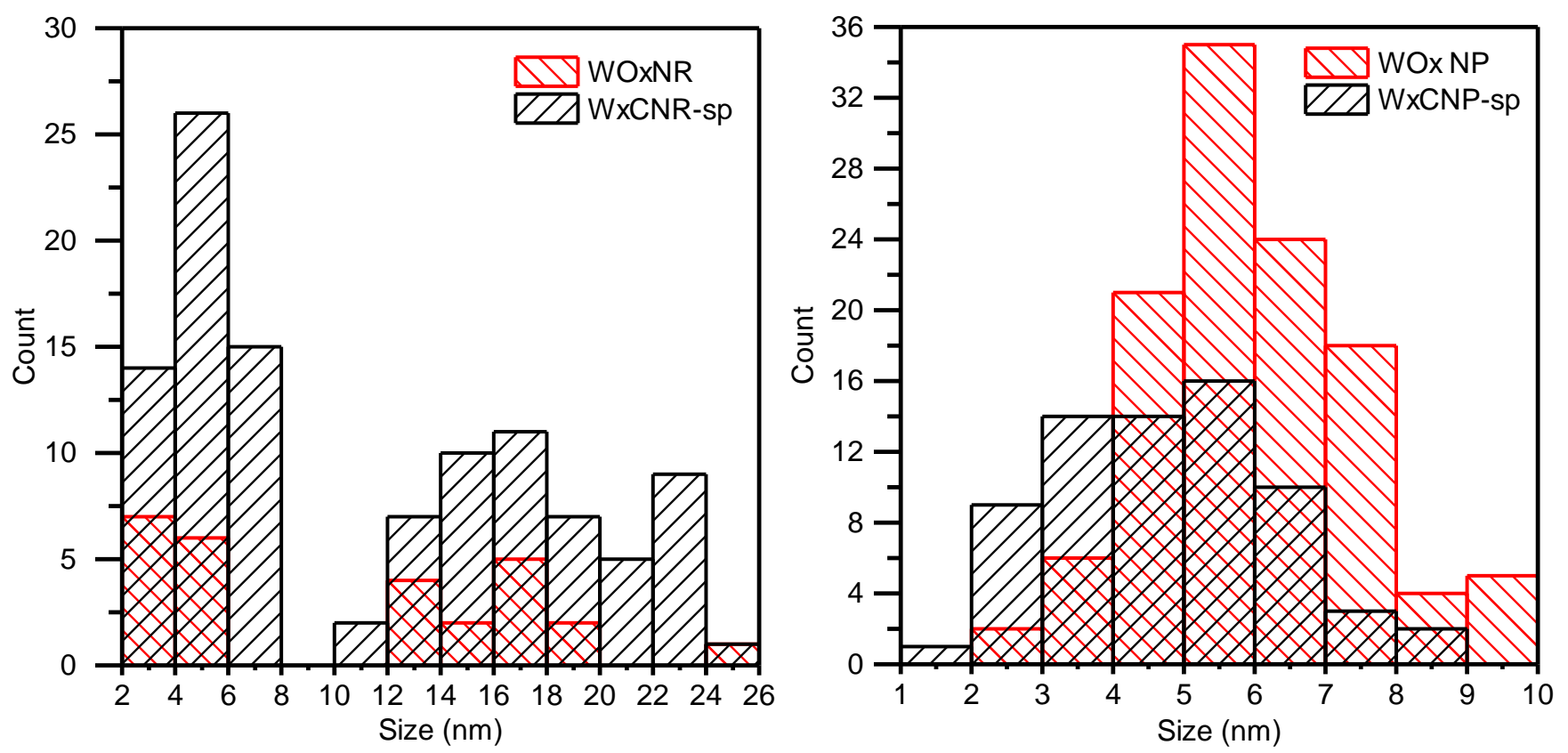

Figure S1. Particle size distributions calculated for nanorod and nanoparticle morphologies for fresh and spent catalysts. Nanorods display a bimodal distribution corresponding to the two measures, length and width, for each nanorod. 


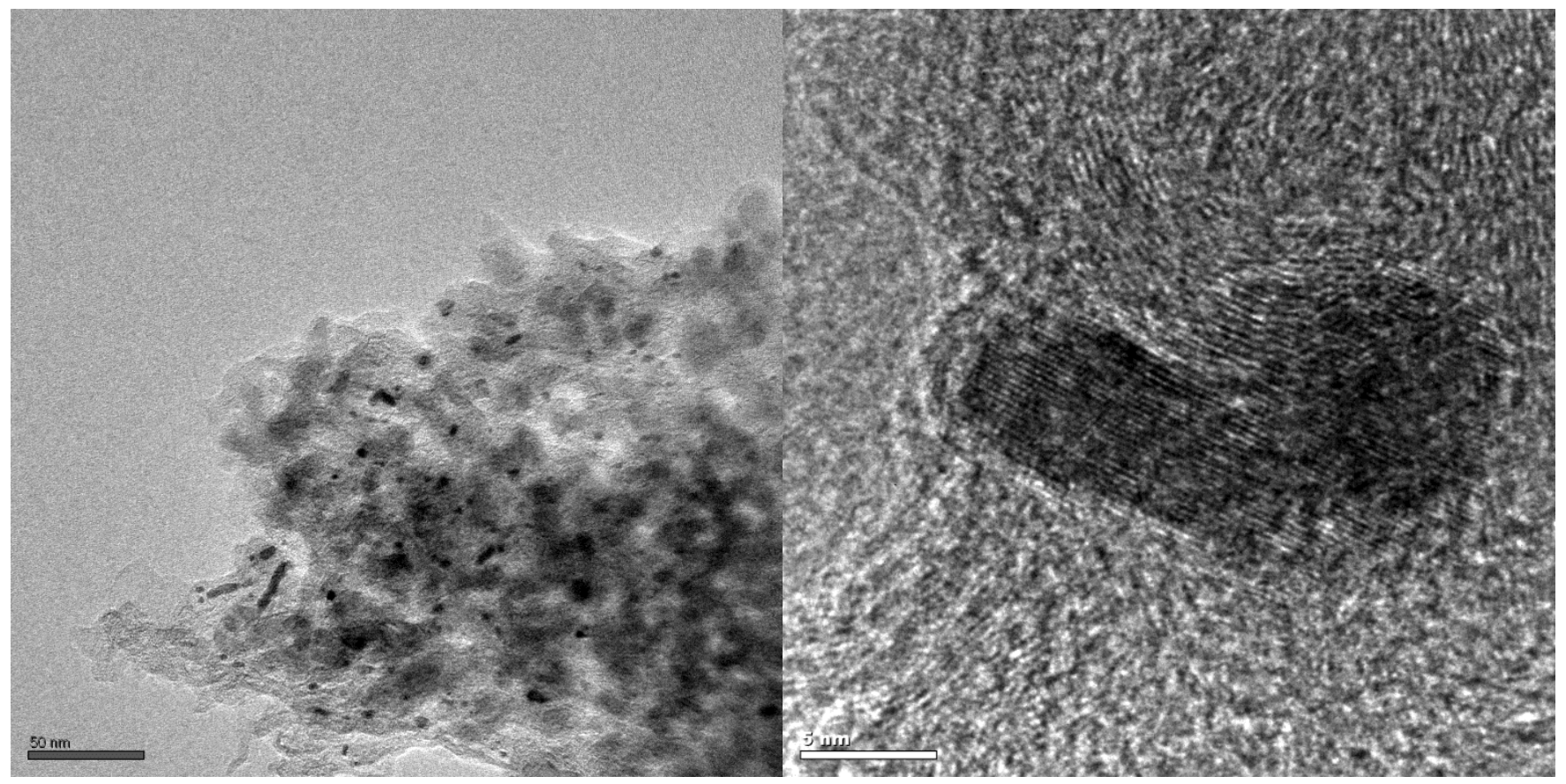

Figure S2. TEM images for (left) $\mathrm{W}_{\mathrm{x}} \mathrm{C}-\mathrm{NP}$-sp and (right) $\mathrm{W}_{\mathrm{x}} \mathrm{C}-\mathrm{NR}-\mathrm{sp}$. $\mathrm{W}_{\mathrm{x}} \mathrm{C}-\mathrm{NP}$-sp did not display coke formations on an imaged sample. $\mathrm{W}_{\mathrm{x}} \mathrm{C}$-NP-sp displays lattice fringes characteristic with graphitic carbon formation.

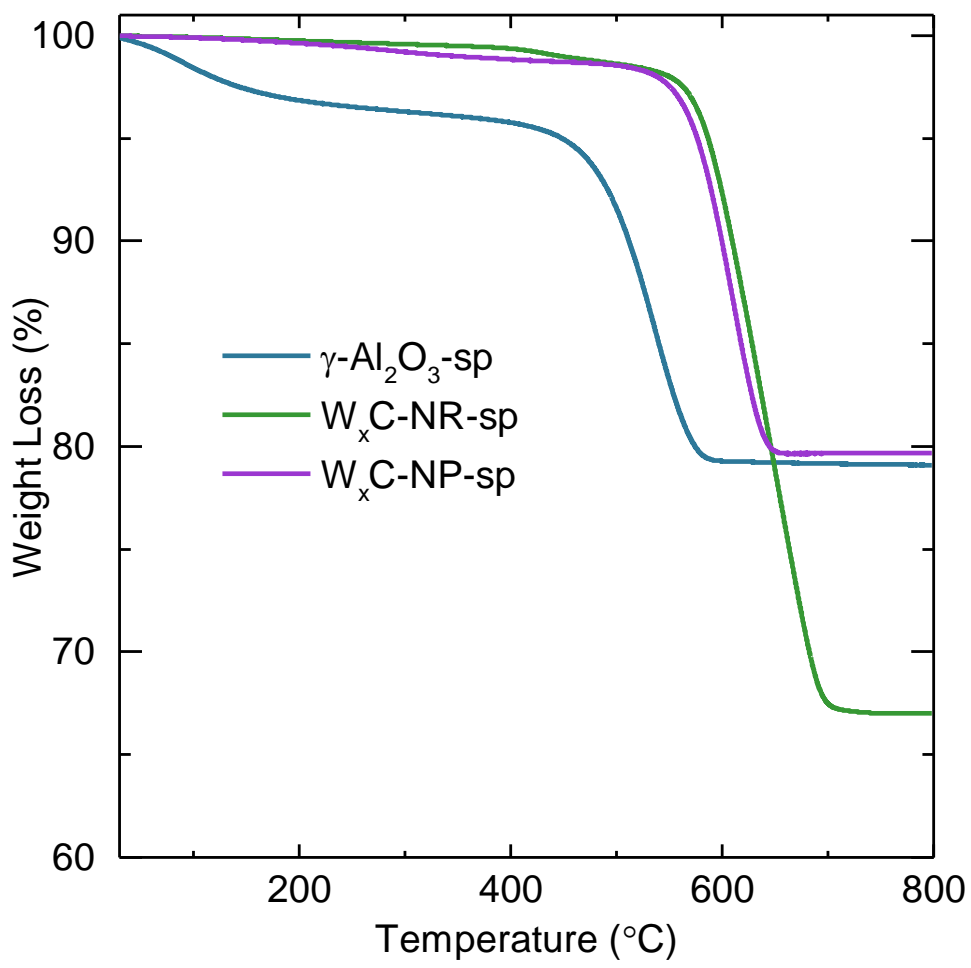

Figure S3. Thermogravimetric analysis (TGA) for $\gamma-\mathrm{Al}_{2} \mathrm{O}_{3}-\mathrm{sp}, \mathrm{W}_{\mathrm{x}} \mathrm{C}-\mathrm{NR}-\mathrm{sp}, \mathrm{W}_{\mathrm{x}} \mathrm{C}-\mathrm{NP}-\mathrm{sp}$ in air to determine to determine the amount of coke formed during reaction for each sample. 
Turnover number (TON) calculation:

$$
\begin{gathered}
\text { Mass of } W_{x} C \text { particle }=\frac{M W W}{6.022 \times 10^{23} \text { atoms }} \times \frac{\# W \text { atoms }}{W_{x} C \text { particle }}+\frac{M W C}{6.022 \times 10^{23} \text { atoms }} \times \frac{\# C \text { atoms }}{W_{x} C \text { particle }} \\
\# W_{x} C \text { particles }=\frac{W_{x} \text { C loading }(g)}{\text { Catalyst }} \times \frac{W_{x} C \text { particle }}{\text { Mass of } W_{x} C \text { particle }(g)} \\
\text { Total mol } W=\frac{\# \text { exposed } W \text { atoms }}{W_{x} C \text { particle }} \times \# W_{x} C \text { particles } \times \frac{1 \text { mol }}{6.022 \times 10^{23} \text { atoms }} \\
\text { Cumulative molar flow }(\mathrm{mol})=\frac{X \text { mL }}{\min } \times \frac{60 \mathrm{~min}}{1 \mathrm{~h}} \times \frac{1 \mathrm{~L}}{1000 \mathrm{~mL}} \times \frac{1 \mathrm{~mol}}{22.4 \mathrm{~L}} \times \text { cumulative time }
\end{gathered}
$$

\begin{tabular}{|l|c|}
\hline Total W atoms (nanorod) & 15616 \\
\hline Total C atoms (nanorod) & 13500 \\
\hline Total W atoms (nanoparticle) & 3439 \\
\hline Total C atoms (nanoparticle) & 1714 \\
\hline Surface W atoms (nanorod) & 4416 \\
\hline Surface W atoms (nanoparticle) & 796 \\
\hline mol W (nanorod) & $2.9 \times 10^{-5}$ \\
\hline mol W (nanoparticle) & $2.44 \times 10^{-5}$ \\
\hline
\end{tabular}

